\title{
Temporal and Spatial Dynamics of Brain Structure Changes during Extensive Learning
}

\author{
Bogdan Draganski, ${ }^{1}$ Christian Gaser, ${ }^{2}$ Gerd Kempermann, ${ }^{3}$ H. Georg Kuhn, ${ }^{4}$ Jürgen Winkler, ${ }^{1}$ Christian Büchel, ${ }^{5}$ and \\ Arne May ${ }^{1,5}$ \\ 1Department of Neurology, University of Regensburg, 93053 Regensburg, Germany²Department of Psychiatry, University of Jena, 07743 Jena, Germany, \\ ${ }^{3}$ Max-Delbrück Center for Molecular Medicine, 13092 Berlin, Germany, ${ }^{4}$ Institute for Neuroscience and Physiology, Gothenburg University, SE 40530 \\ Gothenburg, Sweden, and ${ }^{5}$ Department of Systems Neuroscience, University of Hamburg, 20246 Hamburg, Germany
}

The current view regarding human long-term memory as an active process of encoding and retrieval includes a highly specific learninginduced functional plasticity in a network of multiple memory systems. Voxel-based morphometry was used to detect possible structural brain changes associated with learning. Magnetic resonance images were obtained at three different time points while medical students learned for their medical examination. During the learning period, the gray matter increased significantly in the posterior and lateral parietal cortex bilaterally. These structural changes did not change significantly toward the third scan during the semester break 3 months after the exam. The posterior hippocampus showed a different pattern over time: the initial increase in gray matter during the learning period was even more pronounced toward the third time point. These results indicate that the acquisition of a great amount of highly abstract information may be related to a particular pattern of structural gray matter changes in particular brain areas.

Key words: brain; plasticity; posterior parietal cortex; hippocampus; memory; learning; voxel-based morphometry

\section{Introduction}

One of the most exciting tasks of modern neuroscience is to uncover the functional and structural correlates of learning and memory. Recent theoretical work, as well as neuroimaging and psychological studies, has used a broad spectrum of stimuli to investigate different memory processes. The currently accepted view regarding memory is that items are first kept in the medial temporal lobe system followed by a consolidation process based on changes in the neocortex (Miyashita, 2004) and that regions known to be active during perception and encoding are involved in the subsequent retrieval of learned information (Nyberg et al., 2000; Shannon and Buckner, 2004).

Recent cross-sectional voxel-based morphometry (VBM) studies have demonstrated learning-dependent changes in the adult human brain and suggested anatomical correlates for navigation, arithmetic, linguistic, procedural, and musical learning abilities (Maguire et al., 2000; Golestani et al., 2002; Sluming et al., 2002; Gaser and Schlaug, 2003; Draganski et al., 2004). Given the evidence from a recent longitudinal morphometric study showing that learning a complex visuomotor task induced task-specific transient gray matter changes in the adult human brain (Draganski et al., 2004), we aimed to test the hypothesis whether extensive learning of abstract

\section{Received 0ct. 28, 2005; revised March 28, 2006; accepted April 1, 2006}

We thank all volunteers for their participation in this study and Gerhard Schuierer, Volker Busch, Michael Rose, and Eszter Schoell for technical support. A.M. is supported by Deutsche Forschungsgemeinschaft Grant MA 1862/2. C.B. is supported by Volkswagenstiftung and Bundesministerium für Bildung und Forschung. H.G.K. is supported by VolkswagenStiftung, Vetenskapsrådet, and LUA/ALF Göteborg.

Correspondence should be addressed to Dr. Arne May, Department of Systems Neuroscience, University of Hamburg, Martinistrasse 52, D-22046 Hamburg, Germany. E-mail: a.may@uke.uni-hamburg.de.

DOI:10.1523/JNEUROSCI.4628-05.2006

Copyright $\odot 2006$ Society for Neuroscience $\quad$ 0270-6474/06/266314-04\$15.00/0 information can also induce morphological changes in cortical structures and whether these changes would be transient or long lasting. Based on well established evidence (Eichenbaum, 2004; Squire et al., 2004), we predicted that the medial temporal lobe would show such structural changes.

The German preliminary medical exam, called "Physikum," is usually taken after 2 years at the end of the preclinical education. It includes both oral and written exams in biology, chemistry, biochemistry, physics, human anatomy, and physiology. Consequently, the huge amount of new information, demanding a high level of encoding, retrieval, and usage, requires a 3 month period of daily study sessions and represents an ideal group for investigating possible learning-induced structural plasticity of the adult human brain.

\section{Materials and Methods}

Volunteers

$\mathrm{T}_{1}$-weighted magnetic resonance imaging scans of 38 medical students (21 female, 17 male; mean \pm SD age, $24 \pm 2.3$ years) and 12 age- and sex-matched control subjects ( 8 female, 4 male; mean \pm SD age, $22.1 \pm$ 1.7 years) were performed at two time points [1.5 Tesla Siemens (Munich, Germany) Symphony scanner, magnetization-prepared rapidacquisition gradient echo sequence yielding 150 sagittal slices with a defined voxel size of $1 \times 1 \times 1.08 \mathrm{~mm}]$. In the student group, the first scan was obtained 3 months before the medical exam, and the second scan was performed on the first or second day after the exam. In 23 of these students, a third scan was performed 3 months later. The average grade of our group of volunteers matched the overall average grade of the medical exam that year, which was composed of 7043 medical students, suggesting that our cohort was representative of the population. The control subjects had no exams in the last 6 months and were not studying for any exams at the time. Additionally, they were carefully chosen in regard to educational status (college students for physical therapy) and were scanned at the same first two time points as the medical students. 


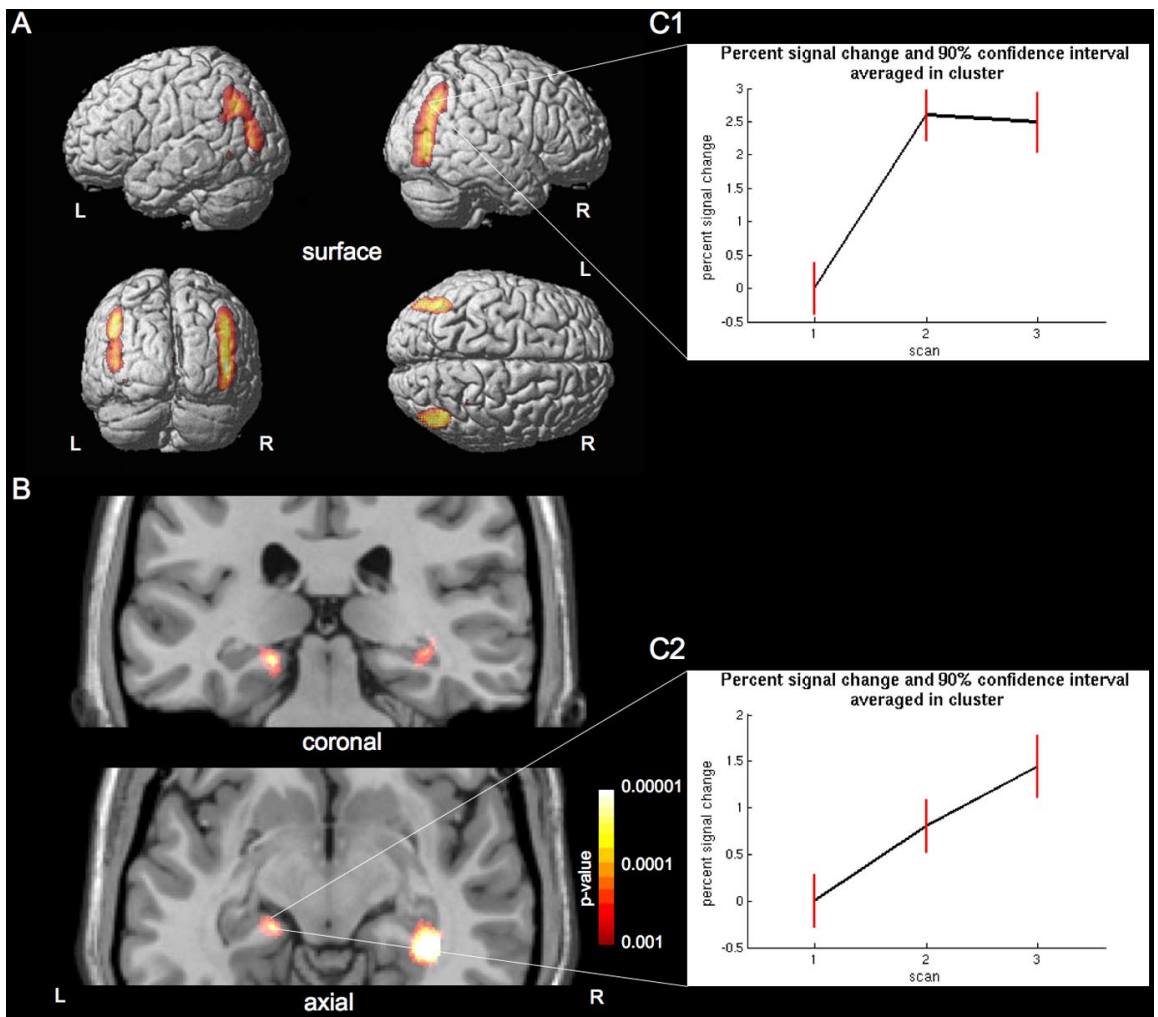

Figure 1. Gray matter increase related to learning. Statistical parametric maps demonstrating the structural difference in gray matter during the learning period in medical students. The left side of the picture is the left side of the brain (L, left; $R$, right). $A$, Significant gray matter increase between the first two time points ( $p<0.05$, corrected for multiple comparisons using FWE) in posterior parietal cortex is superimposed on the cortical surface of a representative single subject. $\boldsymbol{B}$, Significant gray matter increase between the first, second, and third time points in the hippocampus. The image is superimposed ( $p<0.001$ uncorrected) onto two selected slices of a representative single subject. The right hippocampus is significant at $p<0.05$ (FWE corrected), and the left hippocampus is significant after application of a small volume correction using the hippocampal region as defined in the WFU PickAtlas ( $p<0.05$, FWE corrected). C, Plots of percentage of signal change (black line) and $90 \%$ confidence interval (red line) averaged over the cluster of the right posterior parietal cortex (C1) and left hippocampus (C2) over the three time points. The gray matter increase in the parietal cortex had not changed significantly between the second and third scans during the semester break 3 months later. The posterior hippocampus showed an initial increase in gray matter during the learning period, which was even more pronounced in the third scan after the learning period.

The study was approved by the local ethics committee, and written informed consent was obtained from all study participants before examination.

\section{VBM protocol}

VBM is a whole-brain technique that is capable of discovering subtle, regionally specific changes in gray matter by averaging across subjects. VBM has been cross-validated with region-of-interest measurements and functional data in a number of studies (Vargha-Khadem et al., 1998; May et al., 1999; Woermann et al., 1999). This method is based on highresolution structural three-dimensional magnetic resonance images, registered in standard space, and is designed to find significant regional differences throughout the brain by applying voxelwise statistics within the context of Gaussian random fields (Ashburner and Friston, 2000).

The data preprocessing and analysis were performed with SPM2 (Wellcome Department of Cognitive Neurology, London, UK) running under Matlab (MathWorks, Natick, MA). Preprocessing of the data involved spatial normalization, segmentation, modulation, and spatial smoothing with a Gaussian kernel (Ashburner and Friston, 2000). We used a recently described optimized protocol (Good et al., 2001).

\section{Statistical analysis}

Cross-sectional (cohort) analysis. In the cohort analysis, we compared the medical students with the controls at the first time point using a twosample $t$ test to rule out potential differences between the groups.

Longitudinal analysis. The longitudinal analysis for any changes in gray or white matter was performed using a repeated-measures ANOVA and was divided into two parts. In the first analysis, we included the medical students' group over three time points only. We tested for any regions showing an increase in brain structure between the first and second time points. Alternatively, we also tested for a decrease between the first two time points. Furthermore, we tested for regions with an increase between the first, second, and third time point.

In the second analysis, we tested for a change in brain structure between the first and second time points by comparing the medical students with the controls, i.e., a group $\times$ time interaction. We applied a height threshold of $p<0.05$ (corrected for multiple comparisons across the whole brain) and an additional spatial extent threshold (cluster level) of $p<0.001$.

Regression analysis. Additionally, we performed regression analyses using performance and gender as a covariate to test for possible correlations. Again, we applied a threshold of $p<0.05$ (corrected for multiple comparisons across the whole brain).

\section{Results}

Cohort analysis at baseline

The analysis showed no significant regional differences in gray matter between both groups.

\section{Longitudinal analysis: whole-brain correction}

The longitudinal analysis showed a significant increase in gray matter in the parietal cortex, namely in the posterior and inferior parietal lobule bilaterally [right, $(x, y$, $z)(48,-77,6)$, with $t_{(59)}=10.5, p_{\mathrm{FWE}}<$ 0.001 (FWE indicates familywise error rate); left, $(-43,-53,27)$, with $t_{(59)}=$ $\left.7.93, p_{\text {FWE }}<0.001\right]$, which decreased only nonsignificantly toward the third scan (Fig. 1). In concretely looking for areas demonstrating an increase between the first, second, and third scans, the hippocampal increase in gray matter became significant [right, $(38,-35$, $-6)$, with $\left.t_{(59)}=5.71, p_{\mathrm{FWE}}<0.05\right]$ toward the third time point (3 months after the exam).

A decrease in gray matter between the first two time points was found exclusively in the occipito-parietal lobe bilaterally [right, $(18,-90,12)$, with $t_{(59)}=12.42, p_{\mathrm{FWE}}<0.001$; left, $(-13$, $-93,12$ ), with $t_{(59)}=10.46$; $\left.p_{\mathrm{FWE}}<0.001\right]$ (Fig. 2).

Testing for an increase between the first two time points using a group $\times$ time interaction and comparing the medical students and the controls, the above mentioned changes were found exclusively in the medical student group.

\section{Longitudinal analysis: small volume correction}

Our main analysis was performed with $p<0.05$, corrected for multiple comparisons across the whole brain. Using this threshold, we found significant increases in the occipito-parietal lobe bilaterally and in the right hippocampus. Because this very conservative threshold might wrongly suggest lateralization of the hippocampus and considering our a priori hypothesis for the hippocampal area based on previous studies (Maguire et al., 


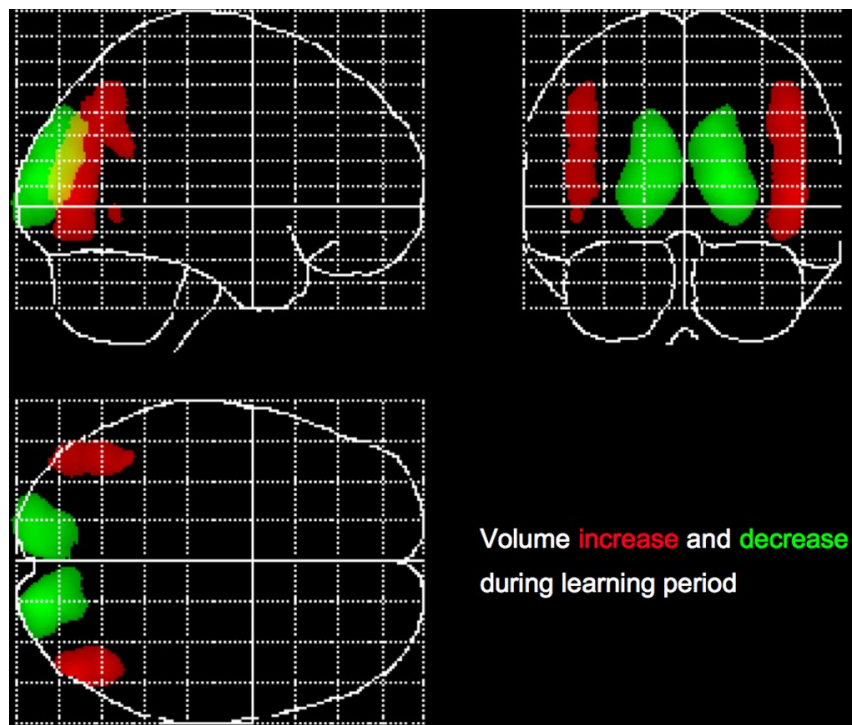

Figure 2. Gray matter increases and decreases displayed as maximum intensity projection. Gray matter increases (red) and decreases (green) are simultaneously shown in a so-called maximum intensity projection, with threshold at $p<0.05$ (corrected for multiple comparisons using FWE).

2000, 2003; Squire et al., 2004), we repeated the same analysis using the hippocampal region as a region of interest. This socalled small volume correction (SVC) was applied with the hippocampal region as defined in the Wake Forest University (WFU) PickAtlas (Maldjian et al., 2003), and we thresholded the result using a $p<0.05$, corrected for multiple comparisons. Using the SVC, additionally to the described changes in the right hippocampus, the left hippocampal area demonstrated also an increase between the first, second, and third scans $[(-19,-29$, $-9)$, with $\left.t_{(59)}=4.74, p_{\mathrm{SVC}}<0.05\right]$.

\section{Regression analysis}

Testing for a correlation between gender or performance on the medical exam and changes in gray matter produced no significant results.

\section{Discussion}

Using voxel-based morphometry, a whole-brain technique that is capable of discovering subtle, regionally specific changes in gray matter by averaging across subjects, we found learning-induced structural changes in the human brain, namely a gray matter increase in the posterior parietal cortex (PPC) and inferior parietal cortex bilaterally. In addition, and in partial contrast to this finding, there was a continuous gray matter increase in the posterior hippocampus throughout the three examined time points, demonstrating an increase even after the learning period. This differentiated temporal and spatial dynamic of morphological brain changes delineates the important role of subsystems in memory formation. We also tested for any decreases in gray matter as well as white matter changes and found a decrease of gray matter between the first and second time points exclusively in the occipital parietal lobe. These changes were, however, directly adjacent to a highly significant increase of white matter in this region, which is in accordance with the literature (Golestani et al., 2002). An increase in white matter volume (i.e., a change of the classification of individual voxels from gray to white matter) will prompt an inverse effect (i.e., regional loss in gray matter volume) in adjacent gray matter. Because no hypothesis exists for a learning-dependant decrease of gray matter, we suggest that these changes are more likely linked to the described (Golestani et al., 2002) regional increase in white matter. These findings have to be viewed with great caution because VBM is not sensitive for detecting changes in white matter.

Consistent with previous neuropsychological studies (Shannon and Buckner, 2004; Wheeler and Buckner, 2004), our results place emphasis on the posterior and inferior parietal cortex in the network of declarative memory. These regions are associated with information transfer into long-term memory (Miyashita, 2004). Recent studies using functional imaging have demonstrated the contribution of the PPC as a key neural locus for storage of visual short-term memory (Linden et al., 2003; Todd and Marois, 2004). Neuroimaging data confirm the involvement of the dorsal and the ventral visual stream in learning and memory, in addition to their role in perception and encoding of visual information (Buchel et al., 1999). Among other brain areas, the PPC is, beyond its role in the processes of space-based attention and motor intention, significantly involved in memory retrieval and memory success (Wheeler and Buckner, 2004).

The gray matter increase in the right posterior hippocampus, known also for its role in semantic and spatial knowledge acquisition (Alvarez and Squire, 1994; Maguire et al., 2003), suggests specific use-dependent plasticity of medial temporal lobe structures. The magnitude of the posterior hippocampal gray matter volume has been shown to correlate positively with spatial representation skills (Maguire et al., 2000). This is probably not innate but depends on the extent of detail and/or duration of use of spatial representations (Maguire et al., 2003). However, the cited morphometric studies compared two cohorts at one time point, depending on post hoc analyses to shed light on the relationship between gray matter differences and experience dependency (Maguire et al., 2003). In this context, it is noteworthy that, beyond its key function as a gate to our long-term memory, the human hippocampus is one of the anatomical structures known for its neurogenesis activity, i.e., the ability to generate neurons derived from local stem cells (Eriksson et al., 1998). Physical activity and an enriched environment, i.e., more opportunity for social interaction, physical activity, and learning, have been shown to improve the rate of neurogenesis and maintenance of these new cells (Kempermann and Gage, 1998; Gage, 2002). Focusing on this issue, we used a regression analysis involving the performance (exam notes) of the students but found no correlation between changes in gray matter and performance. However, we did not control for IQ, learning strategies, or learning hours per day per week (workload), which might have revealed additional differences unrelated to performance.

Because of the limited knowledge regarding the neural code underlying the process of learning, we can only speculate about the nature of the dynamic structural changes. At the cellular level, it has been demonstrated that use-dependent plasticity of synaptic strength and structure is a fundamental mechanism involved in memory encoding (Trachtenberg et al., 2002; Maviel et al., 2004). For both forms of memory storage, a particular synaptic growth is thought to represent the stable cellular change that maintains the long-term process (Bailey et al., 2004). Given that the hippocampus is a well studied brain structure known for gating human long-term memory and being one of the brain areas involved in neurogenesis (Eriksson et al., 1998; Kempermann and Gage, 1998; Gage, 2002), one could argue that the changes seen in the hippocampus in our study may in fact resemble the exciting neural changes seen in rodents (Gould et al., 1999; van Praag et al., 1999, 2002; Shors et al., 2001; Kempermann et al., 2004). 
However, a simple increase in cell size or even a usedependent synaptogenesis (Trachtenberg et al., 2002) would probably have the same impact on our data. One could argue that the morphological changes in the parietal cortex resemble fastadjusting neuronal systems, such as spine and synapse turnover (Trachtenberg et al., 2002), whereas the progressive increase in the hippocampal area involves slow-evolving mechanisms such as neuronal or glial cell genesis (Kempermann et al., 1997). The changes in gray matter volume of the posterior hippocampus past the point of the medical examination is surprising given the fact that the medical students had a break after their exam. One explanation for this phenomenon is that the intense preparation time necessary in preparing for the exam is also a very stressful episode. Intense psychosocial stress has been shown to reduce hippocampal volume and to cause reduced structural plasticity, including dendritic arborization, synapse density, and neurogenesis via elevated corticosteroid levels (McEwen, 1999; Sauro et al., 2003). We can therefore presume that, during the acute study phase, stress-related factors as well as plasticity-enhancing factors were present, whereas after the exam, the continued effect of the cognitive stimulation became more detectable because counteracting stress responses were removed. Moreover, if neurogenesis is one of the contributing factors, we can also anticipate a delay in the increase of gray matter volume, because the generation and full maturation of new neurons in the adult hippocampus requires several weeks to months (Zhao et al., 2006).

Independent of the precise nature of these morphological changes, our results support theoretical considerations stressing structural forms of neuroplasticity to be important for processing information in dynamic networks according to novel informational demands (Chambers et al., 2004). It is reasonable to assume that plasticity is a characteristic of the nervous system that evolved for coping with changes in the environment. Understanding changes in brain structure as a result of learning and adaptation is pivotal in understanding the characteristic flexibility of our brain to adapt.

\section{References}

Alvarez P, Squire LR (1994) Memory consolidation and the medial temporal lobe: a simple network model. Proc Natl Acad Sci USA 91:7041-7045.

Ashburner J, Friston KJ (2000) Voxel-based morphometry: the methods. NeuroImage 11:805-821.

Bailey CH, Kandel ER, Si K (2004) The persistence of long-term memory: a molecular approach to self-sustaining changes in learning-induced synaptic growth. Neuron 44:49-57.

Buchel C, Coull JT, Friston KJ (1999) The predictive value of changes in effective connectivity for human learning. Science 283:1538-1541.

Chambers RA, Potenza MN, Hoffman RE, Miranker W (2004) Simulated apoptosis/neurogenesis regulates learning and memory capabilities of adaptive neural networks. Neuropsychopharmacology 29:747-758.

Draganski B, Gaser C, Busch V, Schuierer G, Bogdahn U, May A (2004) Neuroplasticity: changes in grey matter induced by training. Nature 427:311-312.

Eichenbaum H (2004) Hippocampus: cognitive processes and neural representations that underlie declarative memory. Neuron 44:109-120.

Eriksson PS, Perfilieva E, Bjork-Eriksson T, Alborn AM, Nordborg C, Peterson DA, Gage FH (1998) Neurogenesis in the adult human hippocampus. Nat Med 4:1313-1317.

Gage FH (2002) Neurogenesis in the adult brain. J Neurosci 22:612-613.

Gaser C, Schlaug G (2003) Brain structures differ between musicians and non-musicians. J Neurosci 23:9240-9245.

Golestani N, Paus T, Zatorre RJ (2002) Anatomical correlates of learning novel speech sounds. Neuron 35:997-1010.

Good CD, Johnsrude IS, Ashburner J, Henson RN, Friston KJ, Frackowiak RS (2001) A voxel-based morphometric study of ageing in 465 normal adult human brains. NeuroImage 14:21-36.

Gould E, Beylin A, Tanapat P, Reeves A, Shors TJ (1999) Learning enhances adult neurogenesis in the hippocampal formation. Nat Neurosci 2:260-265.

Kempermann G, Gage FH (1998) Closer to neurogenesis in adult humans. Nat Med 4:555-557.

Kempermann G, Kuhn HG, Gage FH (1997) More hippocampal neurons in adult mice living in an enriched environment. Nature 386:493-495.

Kempermann G, Wiskott L, Gage FH (2004) Functional significance of adult neurogenesis. Curr Opin Neurobiol 14:186-191.

Linden DE, Bittner RA, Muckli L, Waltz JA, Kriegeskorte N, Goebel R, Singer W, Munk MH (2003) Cortical capacity constraints for visual working memory: dissociation of fMRI load effects in a fronto-parietal network. NeuroImage 20:1518-1530.

Maguire EA, Gadian DG, Johnsrude IS, Good CD, Ashburner J, Frackowiak RS, Frith CD (2000) Navigation-related structural change in the hippocampi of taxi drivers. Proc Natl Acad Sci USA 97:4398-4403.

Maguire EA, Spiers HJ, Good CD, Hartley T, Frackowiak RS, Burgess N (2003) Navigation expertise and the human hippocampus: a structural brain imaging analysis. Hippocampus 13:250-259.

Maldjian JA, Laurienti PJ, Kraft RA, Burdette JH (2003) An automated method for neuroanatomic and cytoarchitectonic atlas-based interrogation of fMRI data sets. NeuroImage 19:1233-1239.

Maviel T, Durkin TP, Menzaghi F, Bontempi B (2004) Sites of neocortical reorganization critical for remote spatial memory. Science 305:96-99.

May A, Ashburner J, Buchel C, McGonigle DJ, Friston KJ, Frackowiak RS, Goadsby PJ (1999) Correlation between structural and functional changes in brain in an idiopathic headache syndrome. Nat Med 5:836-838.

McEwen BS (1999) Stress and hippocampal plasticity. Annu Rev Neurosci 22:105-122.

Miyashita Y (2004) Cognitive memory: cellular and network machineries and their top-down control. Science 306:435-440.

Nyberg L, Habib R, McIntosh AR, Tulving E (2000) Reactivation of encoding-related brain activity during memory retrieval. Proc Natl Acad Sci USA 97:11120-11124.

Sauro MD, Jorgensen RS, Pedlow CT (2003) Stress, glucocorticoids, and memory: a meta-analytic review. Stress 6:235-245.

Shannon BJ, Buckner RL (2004) Functional-anatomic correlates of memory retrieval that suggest nontraditional processing roles for multiple distinct regions within posterior parietal cortex. J Neurosci 24:10084-10092.

Shors TJ, Miesegaes G, Beylin A, Zhao M, Rydel T, Gould E (2001) Neurogenesis in the adult is involved in the formation of trace memories. Nature 410:372-376.

Sluming V, Barrick T, Howard M, Cezayirli E, Mayes A, Roberts N (2002) Voxel-based morphometry reveals increased gray matter density in Broca's area in male symphony orchestra musicians. NeuroImage 17:1613-1622.

Squire LR, Stark CE, Clark RE (2004) The medial temporal lobe. Annu Rev Neurosci 27:279-306.

Todd JJ, Marois R (2004) Capacity limit of visual short-term memory in human posterior parietal cortex. Nature 428:751-754.

Trachtenberg JT, Chen BE, Knott GW, Feng G, Sanes JR, Welker E, Svoboda $\mathrm{K}$ (2002) Long-term in vivo imaging of experience-dependent synaptic plasticity in adult cortex. Nature 420:788-794.

van Praag H, Kempermann G, Gage FH (1999) Running increases cell proliferation and neurogenesis in the adult mouse dentate gyrus. Nat Neurosci 2:266-270.

van Praag H, Schinder AF, Christie BR, Toni N, Palmer TD, Gage FH (2002) Functional neurogenesis in the adult hippocampus. Nature 415:1030-1034.

Vargha-Khadem F, Watkins KE, Price CJ, Ashburner J, Alcock KJ, Connelly A, Frackowiak RS, Friston KJ, Pembrey ME, Mishkin M, Gadian DG, Passingham RE (1998) Neural basis of an inherited speech and language disorder. Proc Natl Acad Sci USA 95:12695-12700.

Wheeler ME, Buckner RL (2004) Functional-anatomic correlates of remembering and knowing. NeuroImage 21:1337-1349.

Woermann FG, Free SL, Koepp MJ, Sisodiya SM, Duncan JS (1999) Abnormal cerebral structure in juvenile myoclonic epilepsy demonstrated with voxel-based analysis of MRI. Brain 122:2101-2108.

Zhao C, Teng EM, Summers Jr RG, Ming GL, Gage FH (2006) Distinct morphological stages of dentate granule neuron maturation in the adult mouse hippocampus. J Neurosci 26:3-11. 